LBL --33391

DE93 007662

\title{
AN INTERFACE CONFIGURATION EXPERIMENT ON USML-1*
}

\author{
Paul Concus \\ Lawrence Berkeley Laboratory \\ and \\ Department of Mathematics \\ University of California \\ Berkeley, California 94720 \\ Robert Finn \\ Department of Mathematics \\ Stanford University \\ Stanford, California 94305 \\ Mark Weislogel \\ Space Experiments Division \\ NASA Lewis Research Center \\ Cleveland, Ohio 44135
}

January 1993

\footnotetext{
* This work was supported in part by the Mathematical Sciences Subprogram of the Office of Energy Research, U. S. Department of Energy, under Contract Number DE-AC03-76SF00098, by the National Aeronautics and Space Administration under Grant NAG3-1143, and by the National Science Foundation under Grant DMS89-02831.

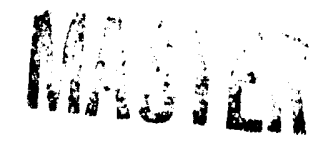

DISTRIBUTION OF THIS DOCUMENT IS UNLIMITEA

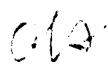




\title{
AN INTERFACE CONFIGURATION EXPERIMENT ON USML-1
}

\author{
Paul Concus* \\ University of California, Berkeley, CA 94720 \\ Robert Finn $\dagger$ \\ Stanford University, Stanford, $C A 94305$ \\ Mark Weislogelł \\ NASA Lewis Research Center, Cleveland, OH 44135
}

\begin{abstract}
Experiments were carried out for "exotic" rotationally symmetric containers aboard the first NASA United States Microgravity Laboratory Space Shuttle flight (USML-1). The containers have the property that they admit an entire continuum of distinct equilibrium rotationally-symmetric capillary free-surfaces for a given liquid volume and contact angle. It was found, after the containers were filled in orbit, that an initial equilibrium interface from the symmetric continuum reoriented, when perturbed, to a stable interface that was not rotationally symmetric, as predicted by the mathematical theory.
\end{abstract}

\section{Introduction}

When planning space-based operations, it is essential to be able to predict the locations and configurations that fluids will assume in containers under low-gravity conditions. For example, one would be in serious difficulty if one did not know in advance in what part of a fuel tank the liquid contents were to be found. Currently available mathematical theory can determine possible free-surface configurations for only a few simple containers, such as the right circular cylinder and the sphere. Even for these geometries, the present theory is inadequate for dealing with such unaccustomed liquid configurations as a column or bridge extending across the interior of the container, a situation that can be expected to occur under reduced gravity.

The classical theory, according to the YoungLaplace-Gauss formulation, characterizes stable fluid configurations as local minima of the surface-plusgravitational mechanical energy. Using this point of view in a mathematical study, we have calculated the shapes of rotationally symmetric "exotic" containers with the remarkable property that for given contact angle and fluid volume, an infinity (in fact, an entire continuum) of distinct rotationally symmet-

* Senior Scientist, Lawrence Berkeley Laboratory and Adjunct Professor, Department of Mathematics

$\dagger$ Professor, Department of Mathematics

$\ddagger$ Aerospace Engineer, Space Experiments Division ric equilibrium configurations can appear, all of which have the same energy (Fig. 1).$^{5,9}$

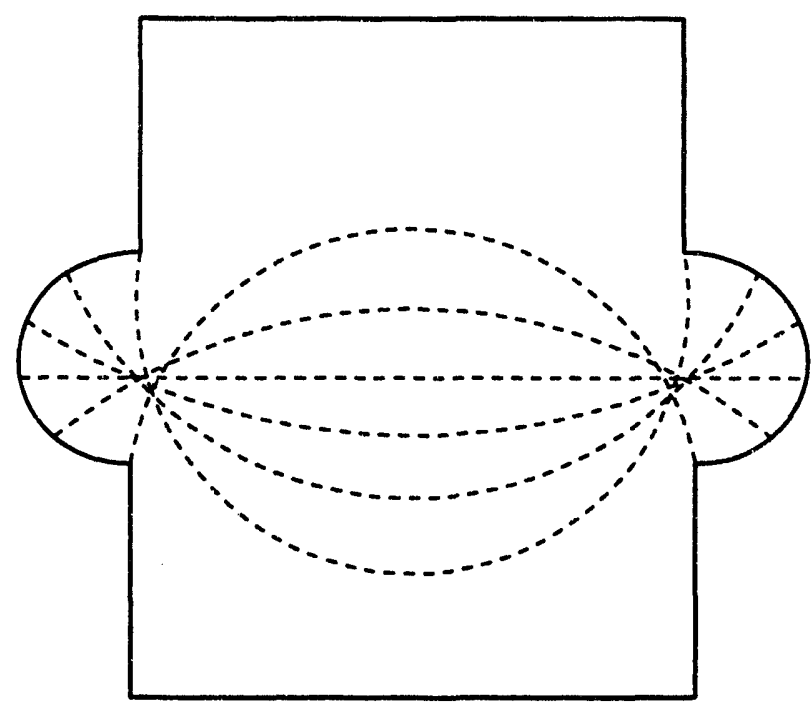

Figure 1. Arial section of an exotic container for contact angle $80^{\circ}$ and zero gravity, depicting meridians (dashed curves) of members of the rotationallysymmetric equilibrium free surface continuum. All surfaces have the same contact angle and energy and enclose the same volume of liquid with the bottom of the container.

These symmetric equilibrium configurations turn out to be unstable, and it can be shown that particular deformations that are not rotationally symmetric yield configurations with lower energy. ${ }^{4,9}$ By a careful construct, it was possible to demonstrate a symmetric container that admits infinitely many symmetric equilibrium interfaces, but for which no interface that minimizes energy can be symmetric. This is in notable contrast with what happens in the familiar case of the right circular cylinder, for which the symmetric interface is stable, and no asymmetric ones can appear. ${ }^{11}$

There is presently no known way to determine mathematically the surfaces that minimize energy. Numerical computations, such as those described in the following section, suggest a number of particular 
non-rotationally-symmetric surfaces as local minima. Additionally, it is not clear to what extent the idealizations of the formal theory will be reflected in reality. Thus it is of interest to determine experimentally what physically observable energy-minimizing configurations there might be for the exotic containers, both as an indication of the limitations of classical theory and as a guide to future theoretical study.

\section{Computed configurations}

According to the classical Young-Laplace-Gauss formulation, the mechanical energy $\mathcal{E}$ of a partly filled container, arising from the associated surface and hydrostatic forces, is (Ref. 8, Chap. 1)

$$
\mathcal{E}=\sigma\left(\mathcal{S}-\mathcal{S}^{*} \cos \gamma\right)+\text { gravitational energy. }
$$

Here $\sigma$ is the liquid-gas interfacial tension and $\cos \gamma$ is the liquid-solid relative adhesion coefficient, where $\gamma$ is the contact angle between the liquid and the container; $\mathcal{S}$ denotes the area of the liquid-gas free surface and $S^{*}$ the area of the portion of the container in contact with the liquid. The quantities $\sigma$ and $\gamma$ depend only on the materials, independent of the container geometry and gravitational field. The classical formulation, upon which our mathematical study is based, does not encompass possible surface friction effects, such as hysteresis and contact line resistance, or thermodynamic Kelvin energy associated with evaporation and condensation.

Configurations of liquid that yield a stationary value for $\mathcal{E}$ subject to the constraint of fixed liquid volume are, according to the classical theory, equilibrium configurations. They will be stable, as well, if $\mathcal{E}$ is locally minimized. In Ref. 5 exotic rotationally symmetric container shapes are calculated from (1) and the requirement that there be a continuum of rotationally symmetric liquid-gas free surfaces all having the same contact angle and enclosing the same volume of liquid with the container. Such container shapes, which were studied first in Ref. 10 for the special case of zero gravity and contact angle $\pi / 2$, can be obtained for any contact angle or gravity level. 5,9

The container shown in Fig. 1 is obtained by placing circular cylindrical extensions with disk ends on a calculated exotic container shape, in this case for zero gravity and contact angle $80^{\circ}$. The dashed curves depict members of the continuum of rotationally symmetric equilibrium interfaces. Although such containers can be constructed for any gravity and contact angle, only under microgravity conditions are they of sufficiently large scale to permit accurate physical experiment and observation. Of principal interest in this study is the case for which gravity is zero, or sufficiently small that the gravitational energy term can be neglected, so that (1) becomes

$$
\mathcal{E}=\sigma\left(S-\mathcal{S}^{*} \cos \gamma\right) \text {. }
$$

In order to obtain an indication of what stable interfaces there might be in an exotic container, such as the one in Fig. 1, numerical calculations were carried out using a modified version of the Surface Evolver program ${ }^{1}$ and the driving software reported in Ref. 2. The Surface Evolver seeks local minima of a discretized energy functional subject to prescribed constraints using a gradient descent method. Surfaces are approximated by a piecewise-linear triangulation, the form of which can be controlled to various degrees with commands available to the user. Under control of the user, the program adjusts the triangulated surface, step-by-step, in an attempt to decrease the energy. From the numerical and graphical output provided by the program, a user interprets whether a local minimum has been found.

Results obtained with the Surface Evolver for the container depicted in Fig. 1 for zero gravity and contact angle $80^{\circ}$ are shown in Fig. 2. Similar results are shown in Fig. 3 for an exotic container and liquid with $\gamma=55^{\circ}$. (The values $55^{\circ}$ and $80^{\circ}$ correspond to the materials used in the USML-1 experiment.) The surfaces shown are the lowestenergy ones found using the Surface Evolver, starting from the initial horizontal planar member of the symmetric equilibrium interface continuum, to which a small volume-preserving perturbation had been added. Other local minima could be found $b$, varying the form of the initial perturbation, but these had greater energy, although still less than that for the rotationally symmetric surface continuum. ${ }^{2}$ The minimizing free surface is shown as a lighter surface against the darker, checked background of the container; both have been shaded by the (color) graphics display software. Triangulation mesh lines are in white. To expose the free surface to view, only half the container, lying to one side of a vertical plane through its axis of symmetry, is shown. This vertical plane is one about which the free surface possesses reflective symmetry. The exotic, bulge portion of the container and a small length of the circular cylindrical extensions are depicted. Views for the upper figures are from a point on a horizontal plane through the maxinual width of the bulge; the vertical symmetry plane of the free surface is in the plane of the paper. The liquid would lie below and to the left of the free surface. For the lower figures the viewpoint is slightly above the maximal-bulge plane and to the right of the one for the upper figures, to show the excursion of the surface across the bulge portion of the containers.

Both the $55^{\circ}$ and $80^{\circ}$ minimal-energy surfaces appear to be pinned over a part of their circumference to the join between the bulge and lower cylinder portion of the container. The $80^{\circ}$ surface, after traversing the bulge, appears pinned to the upper join 

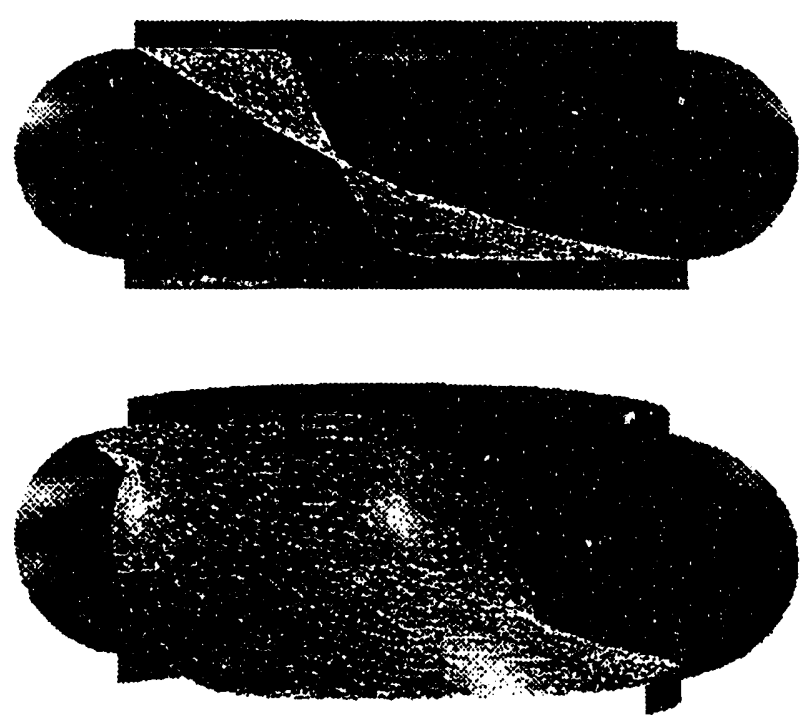

Figure 2. Views of calculated energy-minimizing equilibrium interface in an exotic container. Zero gravity, $\gamma=80^{\circ}$.

as well. This pinning to the upper join could be attributed, in part, to the larger fluid volume for this case; the fluid corresponding to the planar equilibrium symmetric surface with the appropriate contact angle rises higher in the bulge of the $80^{\circ}$ container than in the $55^{\circ}$ one.

These mathematicai and computational predictions are of central interest for the USMI-1 experiment, which we discuss in the remainder of the paper. The experiment confirms these predictions derived from the idealized classical theory and indicates also the role of surface resistance forces in creating a barrier that must be overcome, in moving from the symmetric equilibrium configuration into asymmetric configurations of lower potertial energy.

\section{Experimental apparatus}

The vessels for the Interface Configuration Experiment (ICE), one of which is depicted in Fig. 4, were fabricated at the NASA Lewis Research Center. Each weighs about $1.5 \mathrm{Kg}$, including the liquid contents, and measures approximately $9 \times 9 \times 18.4 \mathrm{~cm}$. The interior was bored out of a solid rectangular block of acrylic plastic, to limit optical distortion. The coordinates for the bulged portion of the container were fed into a numerically controlled air bcaring lathe, which performed the final machining operations. The surfaces were then lightly finished with cloth and a polishing compound. After polishing and annealing, the interior surface deviated less than $50 \mu \mathrm{m}$ from the specified, calculated one.

The vessels were designed for use in the USMI-1
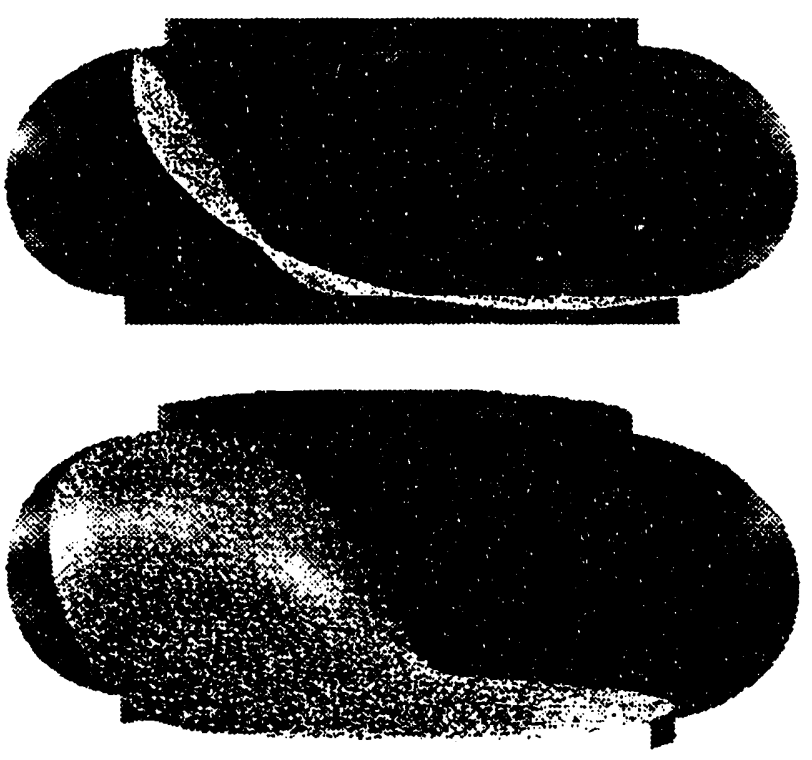

Figure 3. Views of calculated energy-minimizing equilibrium interface in an exotic container. Zero gravity, $\gamma=55^{\circ}$.

Glovebox Experiment Module, which was provided by the European Space Agency/ESTEC. The Glovebox is a multi-user facility developed for experiments to be conducted on Spacelab missions beginning with USML-1. The purpose of the Glovebox is to provide a work area that is ergonomically sound and will allow a payload specialist to carry out operations using small quantities of toxic, irritant, or potentially infective materials, which must be prevented from contaminating the spacecraft atmosphere. Previously designed to handle biological experiments, the Glovebox has been adapted to handle fluids, combustion, and materials science experiments, to permit effective use of hands-on interaction by the payload specialists. ICE utilized the Glovebox primarily as a staging area and a level of containment in the event of fluid leaks. ICE was one of fifteen Glovebox experiments aboard the NASA Space Shuttle Columbia on USML-1 (STS- 50), launched in June 1992.

\section{Experiment description}

The general experimental procedure for ICE during the USML-1 flight was to partly fill the selected vessels with prescribed volumes of fluid and to record with two video cameras the fluid interface configurations that resulted.

Four vessels were fabricated for the tests. They are similar in construction to the vessel shown in Fig. 4. The primary vessel components are the singlepiece acrylic-plastic (transparent) body, an aluminum piston and control dial, stainless steel drive screw and two-port valve, and magnetized feet for securing 


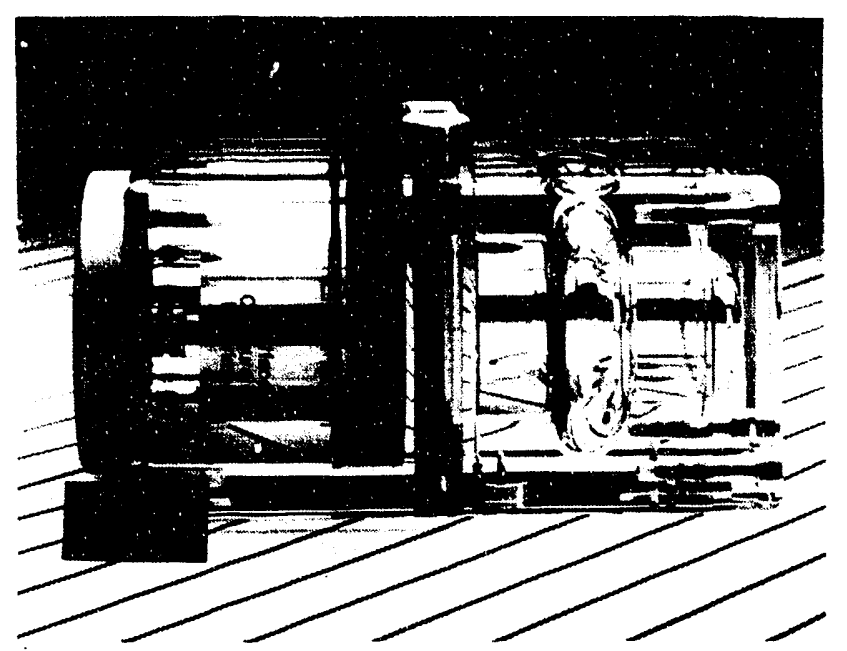

CBB 920-10037

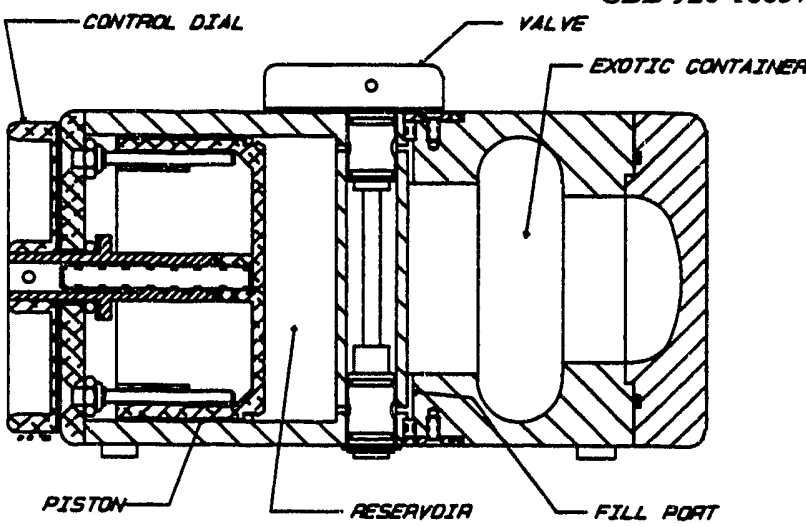

Figure 4. Flight module and schematic drawing of an interface configuration experiment vessel. Exotic container is for zero gravity and $\gamma=55^{\circ}$.

the vessel to the Glovebox labjack. As indicated in Fig. 4, the vessel is placed in the Glovebox in a horizontal position. One of the test fluids is an "immersion" fluid that has a refractive index matched with that of the acrylic container, for reduction of optical distortions; it is a blend of hydrogenated terphenyl and an aliphatic hydrocarbon. Two of the vessels contained this fluid. The interiors of these vessels were coated with surface modifier FC-723 to produce a desired contact angle. Distilled water was used in the other two containers, which were not coated.

The fluids were lightly dyed in order to enhance visibility of the free surface. For the immersion fluid a ceres red dye was used, and for the water a blue food coloring was used, which was an aqueous solution of propylene glycol with propylparaben and sodium metabisulfite as preservatives. Both concentrations were so small that no measurable effects were detected on the values of surface tension or contact angle after the dye was introduced. Surface tension was measured by the Dunuoy ring method as $72.4 \mathrm{dyne} / \mathrm{cm}$ for the water and 32.4 dyne $/ \mathrm{cm}$ for the immersion fluid, with a repeatable accuracy of $\pm 0.2 \mathrm{dyne} / \mathrm{cm}$. The wedge method (Ref. 3, pp. 220-221), (Ref. €, pp. 191-192), was used for measuring the contact angle of water with the acrylic plastic as $80^{\circ}$ with a repeatable accuracy of $\pm 2^{\circ}$. For the immersion fluid, a modified sessile drop method yielded a contact angle with the coated surface of approximately $55^{\circ}$. This method is more sensitive to hysteresis effects; the accuracy is estimated at $\pm 4^{\circ}$.

The interior surfaces of the vessels were cleaned with sequential rinses of a strong ethanol/distilled water solution and distilled water. The vessel was then allowed to dry in a pure nitrogen clean-room environment. Before being injected into the vessel reservoirs, the fluids were filtered with P4 filter paper. The injection was carried out in the nitrogen environment. All seals on a vessel were lubricated with the particular fluid used for it, to eliminate possible contamination from other lubricants.

The diagnostics for the tests included two fullcolor 1:1 video cameras to record the fluid interface configurations, a Glovebox video camera with audio, and devices for the measurement of ambient Glovebox temperature and local acceleration levels.

\section{Experiment procedure}

The crew procedures for carrying out ICE consisted of six steps: (1) unstow equipment, (2) set up Glovebox and install vessel, (3) fill vessel test volume with fluid, (4) observe equilibrium interfaces, (5) disturb interface configuration(s) to determine stability, (6) reverse fill procedure and stow. Up to 60 minutes was required for each vessel when it was tested independently. Less time was necessary when the vessels were tested sequentially.

To begin the experiment, a crew member unstowed the labjack, video cameras, multi-use arm and clamp, and the ICE vessel to be tested. The Glovebox power was switched on and the ICE vessel was placed (horizontally) on the labjack. One video camera was mounted to the front door of the Glovebox, and the other was directed down from the top, secured by the multi-use arm and clamp. The field of view for the cameras was centered on the bulge portion of the exotic container. Iterative adjustments of the cameras and vessel were necessary to center the field properly for both cameras. The Glovebox doors were then replaced with cuff attachments, and the cameras were turned on to record the interface configuration.

To carry out the fill procedure, the crew member opened the quarter-turn valve and turned the control dial on the vessel, displacing the entire fluid contents of the reservoir slowly into the exotic container. The volume of fluid corresponded to that for the symmetric family of equilibrium interfaces, as calculated for the planar member of the family (Fig. 1). Time 
was then allowed for the fluid configuration to stabilize fully while being recorded by the cameras. Five minutes were allowed for the filling procedure. The crew member then disturbed the surface by tapping the container with a finger, lightly at first and then subsequently with moderately increasing force. New surfaces that formed in the container during the tapping procedure were given time to stabilize and to be captured on video. The tapping continued until the surface either broke up or consistently returned to a particular configuration, at which point the fill procedure was to be reversed to empty the exotic container test volume. The vessel and support equipment were then stowed.

\section{Postflight data analysis}

The principal data for this experiment are the video recordings of the two orthogonal cameras. The Glovebox temperature, VCR audio, and the Space Acceleration Measurement System (SAMS) provide additional data, which are useful for interpreting the video observations.

When the on-board video recordings have been obtained by the authors (they have not yet been released by NASA), quantitative data reduction can be attempted directly for the two vessels containing the immersion fluid. Because the optical properties of this fluid are the same as those of the acrylic plastic, the two orthogonal views of the video cameras, once scaled, may be digitized directly; it may then be possible to combine them to reconstruct the full three-dimensional configurations. From such reconstruction, interfacial energies can be estimated as well as apparent contact angles. These parameters are important for comparison with numerical predictions.

The two immersion-fluid-filled vessels are identical, being constructed for the $55^{\circ}$ contact angle measured for the materials; the second provides a repeat run for experiment control purposes. The third vessel, which contained distilled water, was constructed for the contact angle of $80^{\circ}$. A similar vessel was tested previously in the NASA Lewis Research Center Zero Gravity Facility five-second drop tower. ${ }^{7}$ Although insufficient low-gravity time was available for a stable interface to be achieved, observations showed reorientation toward a particular asymmetric configuration. The $80^{\circ}$ vessel tested on USML-1 allows comparison with these ground-based test results, and, more importantly, for obtaining information on the behavior resulting from disturbing the surfaces that form in space.

The fourth vessel also contained distilled water as test fluid, yet it differs from the others in that the container is not exotic; the bulge is a portion of a sphere, for which complete mathematical results are available. The infinite family of distinct rotationally symmetric equilibrium interfaces that are possible for the exotic containers cannot form. However, by rigidbody rotation of the liquid, an equilibrium surface in zero gravity can be tilted as desired with respect to the symmetry axis, without any change in shape or energy. The results for this vessel can act as a control to compare with the behavior in the exotic containers, and for obtaining information about the effects on surface reorientation of resistance at the contact line and presence of the (reentrant) corner between the bulge and cylindrical portions of the containers.

Although the video recordings taken on board USML-1 have not yet been released, some excerpts were downlinked to earth during the flight; from these and from comments made by the payload specialists, preliminary remarks can be made. In all cases, the displacement of liquid from the reservoir to the container was reported as being accomplished successfully. The downlinked video images indicate that at the end of the filling procedure the free surface was in accordance with one that was predicted: a spherical cap corresponding to a particular member of the continuum of rotationally symmetric (unstable) equilibrium interfaces. The liquid then moved to an asymmetric configuration in the each of the three exotic containers after it was tapped by the payload specialist. This was in accordance with mathematical predictions, and the video views of the shapes appear to be in agreement with computed lowest energy configurations (Figs. 2 and 3). The asymmetric configuration behaved very stably in response to subsequent induced disturbances. No configurations resembling those from other computed local-minimum energies at higher energies were observed. The interface in the control vessel did not exhibit the type of reorientation described above; this, too, is in accordance with theory.

Corroboration and quantitative evaluation of the results, as well as further assessment of effects of hysteresis and other factors, await complete analysis of the data. Some video images downlinked to earth during the flight will be shown during the oral presentation, to indicate fluid behavior in one of the immersion-fluid-filled exotic containers.

\section{Concluding Remarks}

The Interface Configuration Experiment (ICE) on the first NASA United States Microgravity Laboratory (USML-1), launched in June 1992, explored a striking behavior of liquid-vapor interfaces that has been predicted mathematically for certain "exotic" containers in a low-gravity environment. Preliminary results from downlinked video images show that an initial equilibrium interface from the rotationally symmetric continuum reoriented, when perturbed, to a stable interface that was not rotationally symmet- 
ric, as predicted by the idealized mathematical theory. The results indicate also the role of contact-line resistance forces (contact-angle hysteresis) in creating a barrier that must be overcome, in moving from the symmetric equilibrium configuration into asymmetric configurations of lower potential energy. More detailed and extensive tests are expected to yield further information on the significance of the findings and on sensitivity to effects not included in the present theory, such as those associated with contact-line resistance and with inaccuracies in measuring contact angles and in fabricating the vessels. The present and future experiments can contribute to determining the applicability of the existing theory and the ability of the theory to predict fluid interfacial configurations for arbitrary container geometries.

\section{Acknowledgments}

We wish to thank V. Brady and M. Callahan for numerical determination of local energy minima and preparation of the graphical images shown in Figs. 2 and 3; D. Gotti and the machine shops at the NASA Lewis Research Center for their role in designing and fabricating the flight units; and the USML-1 crew and NASA staff, whose impressive commitment and expertise made the experiment possible. This work was supported in part by the National Aeronautics and Space Administration under Grant NAG3-1143, by the National Science Foundation under Grant DMS89-02831, and by the Mathematical Sciences Subprogram of the Office of Energy Research, U. S. Department of Energy, under Contract Number DE-AC03-76SF00098.

\section{References}

${ }^{1}$ K. Brakke, Surface Evolver program, avail- able by anonymous ftp from geom.umn.edu as file evolver.tar. $Z$ in directory pub, The Geometry Center, 1300 So. 2nd St., Minneapolis, MN.

${ }^{2}$ M. Callahan, P. Concus, and R. Finn, Energy minimizing capillary surfaces for exotic containers, in Computing Optimal Geometries (with accompanying videotape), J. E. Taylor, ed., AMS Selected Lectures in Mathematics, Amer. Math. Soc., Providence, RI, 1991, pp. 13-15.

${ }^{3} \mathrm{P}$. Concus and R. Finn, On capillary free surfaces in a gravitational field, Acta Math. 132 (1974), pp. 207-223.

${ }^{4} \mathbf{P}$. Concus and R. Finn, Instability of certain capillary surfaces, Manuscr. Math. 63 (1989), pp. 209-213.

${ }^{5}$ P. Concus and R. Finn, Exotic containers for capillary surfaces, J. Fluid Mech., 224 (1991), pp. 383-394; Corrigendum, J. Fluid Mech., 232 (1991), pp. 689-690.

${ }^{6} \mathrm{P}$. Concus and R. Finn, Capillary surfaces in microgravity, in Low-Gravity Fluid Dynamics and Transport Phenomena, J. N. Koster and R. L. Sani, eds., Progress in Astronautics and Aeronautics, Vol. 130, ALAA, Washington, DC, 1990, pp. 183-206.

${ }^{7}$ P. Concus, R. Finn, and M. Weislogel, Droptower experiments for capillary surfaces in an exotic container, AIAA J., 30 (1992), pp. 134-137.

${ }^{8}$ R. Finn, Equilibrium Capillary Surfaces, Springer-Verlag, New York, 1986.

${ }^{9}$ R. Finn, Nonuniqueness and uniqueness of capillary surfaces, Manuscr. Math. 61 (1988), pp. 347-372.

${ }^{10}$ R. Gulliver and S. Hildebrandt, Boundary configurations spanning continua of minimal surfaces, Manuscr. Math., 54 (1986), pp. 323-347.

${ }^{11}$ T. I. Vogel, Uniqueness for certain surfaces of prescribed mean curvature, Pacific J. Math., 134 (1988), pp. 197-207. 

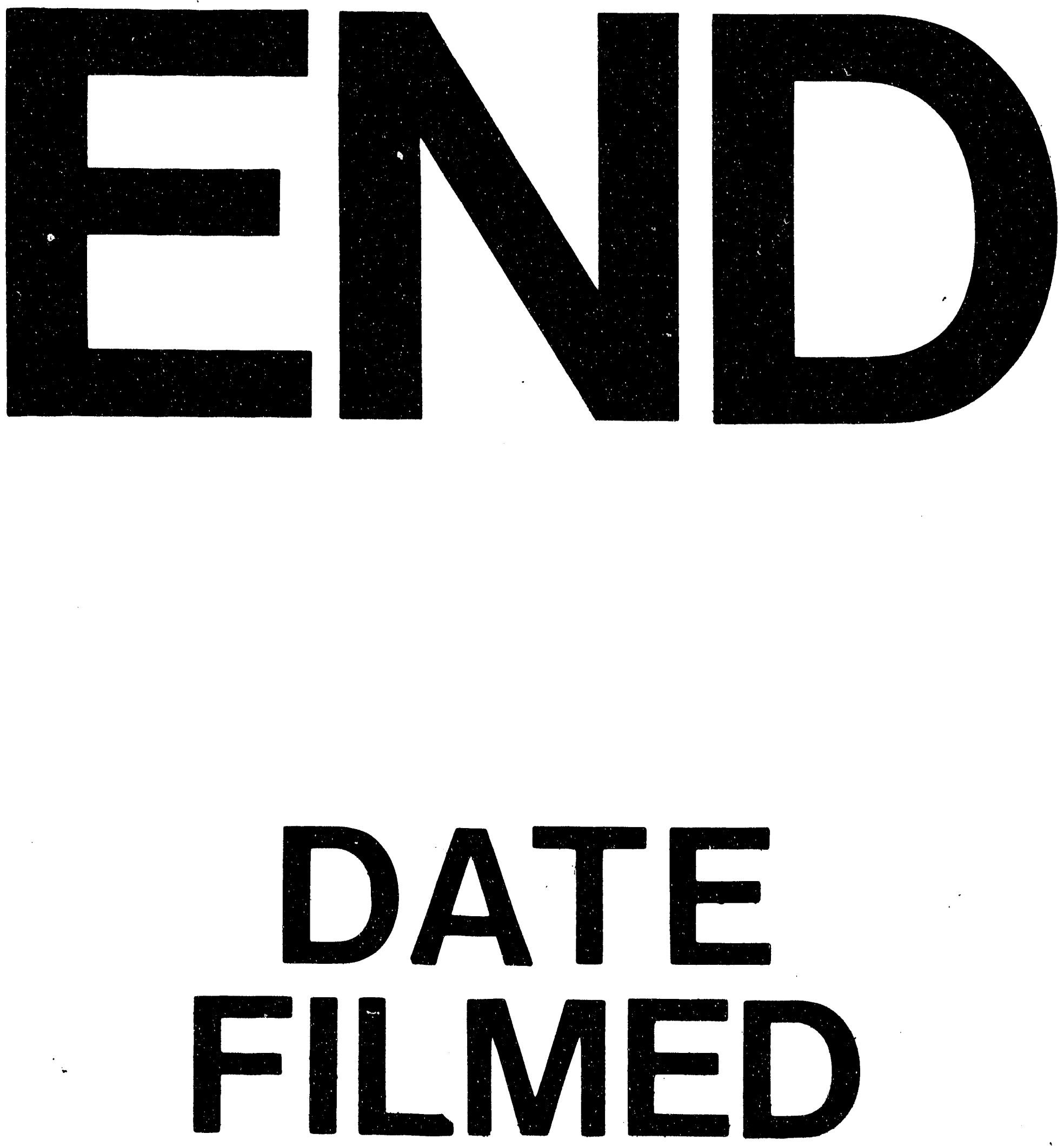

4
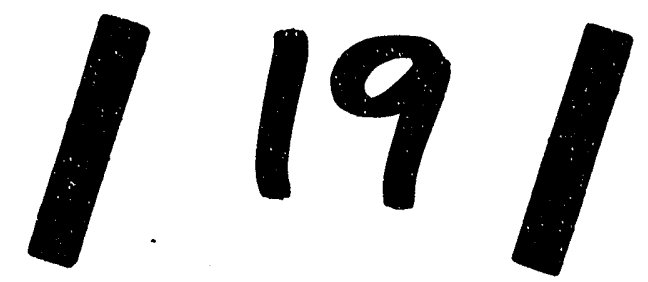

93 
